\title{
Dynamic visceral ischaemia in type A dissection
}

\author{
Francesco Torella ${ }^{1}$, Ahmed Othman ${ }^{2}$, and Manoj Kuduvalli ${ }^{2}$ \\ ${ }^{1}$ Liverpool University Hospitals NHS Foundation Trust \\ ${ }^{2}$ Liverpool Heart and Chest Hospital NHS Foundation Trust
}

December 20, 2020

\begin{abstract}
We describe a case of dynamic obstruction of the mesenteric arteries caused by aortic dissection. The flow pattern demonstrates a periodic flow variation, during which peak systolic velocity varied fourfold.
\end{abstract}

\section{Dynamic visceral ischaemia in type A dissection}

*Francesco Torella ${ }^{1,2}$

Ahmed A Othman ${ }^{1}$

Manoj Kuduvalli ${ }^{1}$

${ }^{1}$ Liverpool Cardiovascular Surgery and ${ }^{2}$ Liverpool Centre for Cardiovascular Science, Liverpool Heart and Chest Hospital, Liverpool, L14 3PE, UK.

*corresponding author

Funding: none

Conflicts of interest: none

Data availability: NA

Informed consent and ethical approval were waived for this report, which contains no patient identifiable data.

A 59-year-old man was referred with Stanford type-A (DeBakey type-I) aortic dissection, abdominal pain and reduced perfusion of the left leg. Lactate levels were elevated $(7.5 \mathrm{mmol} / \mathrm{L})$ and, on CT, the true aortic lumen at the visceral segment appeared compromised (Figure 1). A sternotomy was performed and extended as a laparotomy to a point mid-way between the xyphoid process and the umbilicus. The bowel appeared viable, with poor peristalsis; the hepatic artery and the superior mesenteric artery (SMA) demonstrated weak/intermittent pulsation. Both were interrogated with Doppler (MiraQ, Medistim, Nottingham, UK): flow velocity appeared variable, with a distinctive periodic cycle lasting five pulsations (figure 2). The patient underwent hemiarch repair. On completion, the bowel was still viable with good peristalsis and strong pulsation in the visceral arteries. Post-operatively, femoral pulses were palpable; lactate levels normalised. The patient tested positive for COVID-19 but recovered uneventfully, aside for a peri-pancreatic collection detected on $\mathrm{CT}$, which required no intervention.

This case sheds light on the physiopathology of ischaemia due to functional occlusion of aortic branches in acute dissection. It is widely known that pressure in the false lumen can obliterate aortic branches either by flattening the septum against their origins or by extending the dissection along their course. Intuitively, one may assume that dynamic occlusion occurs periodically during a single cardiac cycle. Our findings, 
however, demonstrated a more complex pattern, with a period of five cycles, during which peak velocity varied fourfold.

The case also highlights the pitfalls of investigating (dynamic) acute dissection with static imaging modalities. Due to its high resolution and image acquisition speed, CT is considered the gold-standard, ${ }^{1}$ although it can only visualise contrast in the aortic lumen(s) at a specific point in time. MRI/MR angiography does not have this limitation and is emerging as an alternative technique for dynamic aortic imaging in acute aortic syndromes. ${ }^{2}$ In our case, extemporaneous use of Doppler equipment normally used to interrogate coronary grafts provided dynamic information.

Finally, the case confirms that obliteration of the false lumen at the entry tear usually results in resolution of dynamic ischaemia. In this patient, the high lactate and his symptoms/signs led us to inspect the bowel due to a high suspicion of impending intestinal gangrene. We were prepared to directly perfuse the SMA and/or the coeliac axis on-pump during the hemiarch repair. Fortunately, the findings at laparotomy were reassuring and the patient was able to undergo a proximal repair without major complications.

\section{References}

Richter GM. State-of-the-Art Imaging of the Aorta with Respect to Endoluminal Intervention. Cardiovasc Intervent Radiol 2020;43:1745-1755.

Bossone E, Czerny M, Lerakis S, et al. Imaging and Biomarkers in Acute Aortic Syndromes: Diagnostic and Prognostic Implications. Curr Probl Cardiol 2020 Jul 22:100654. doi: 10.1016/j.cpcardiol.2020.100654.

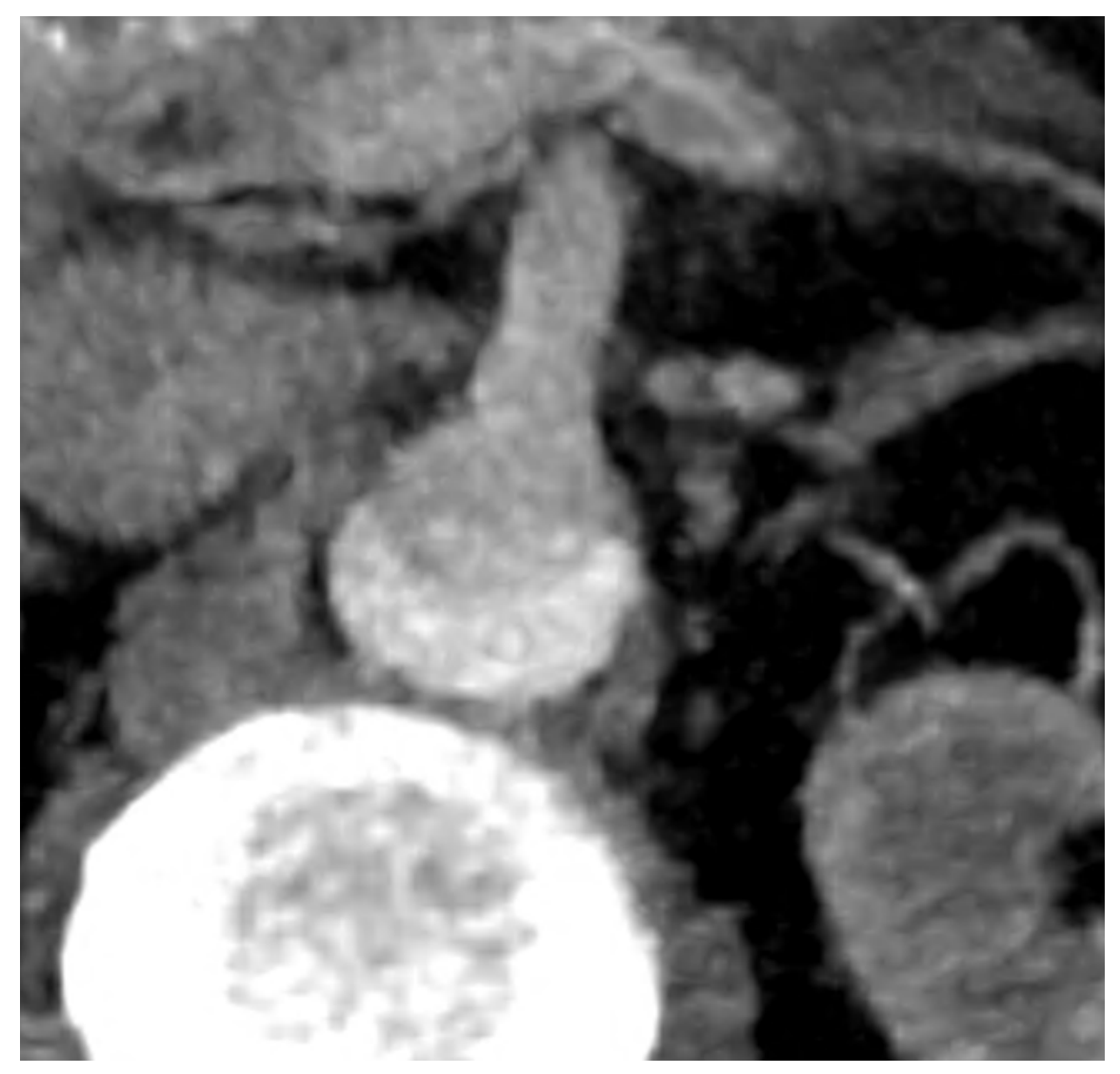




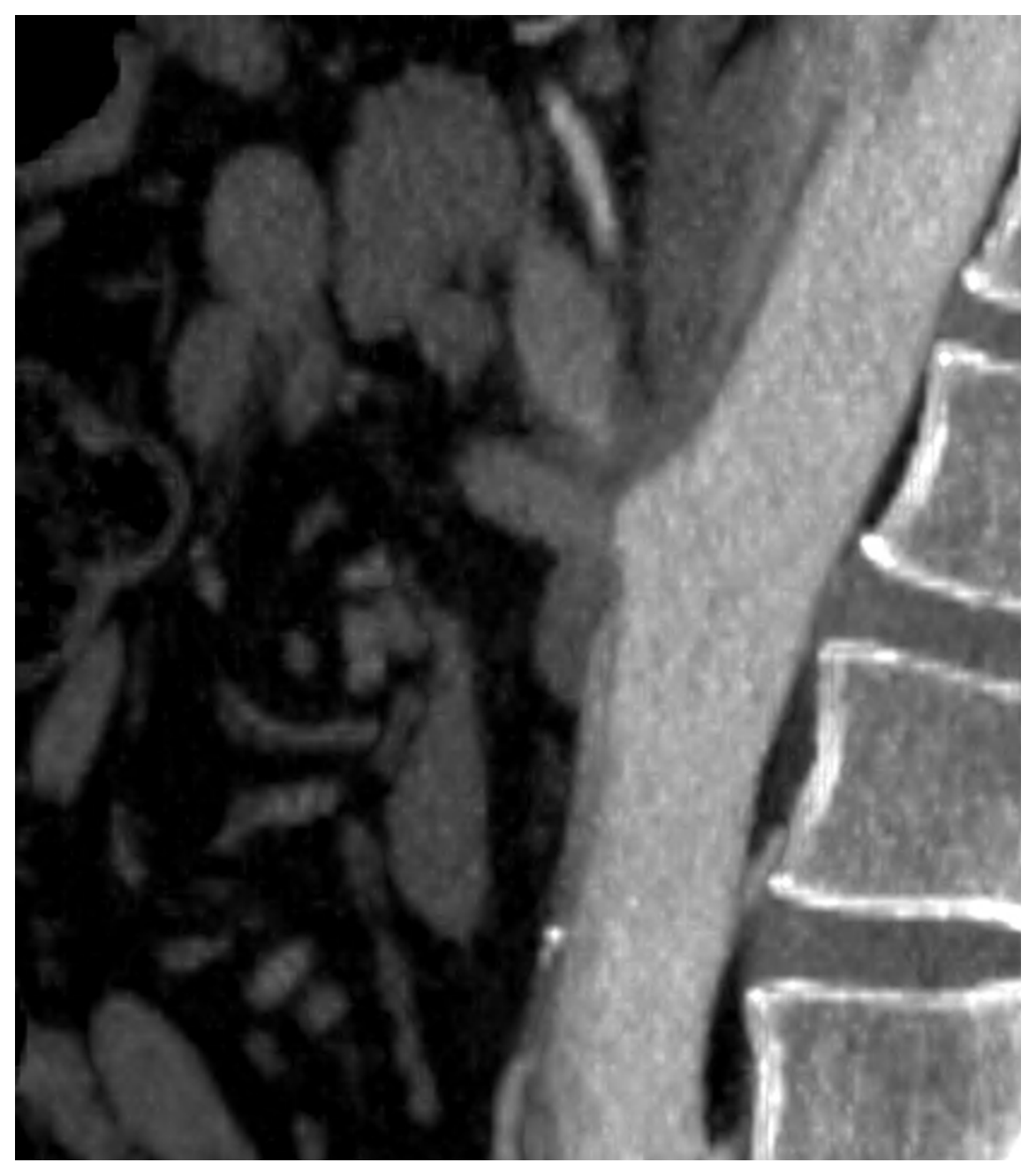

Figure 1

Reconstructed sagittal and axial CT images. Pre-operatively (a), the true lumen (arrow) appeared severely compressed by the false lumen (FL); there was poor enhancement of the coeliac and superior mesenteric arteries. Post-operatively (b), there was partial re-expansion of the true lumen, with improved enhancement of the visceral vessels.

Figure 2 


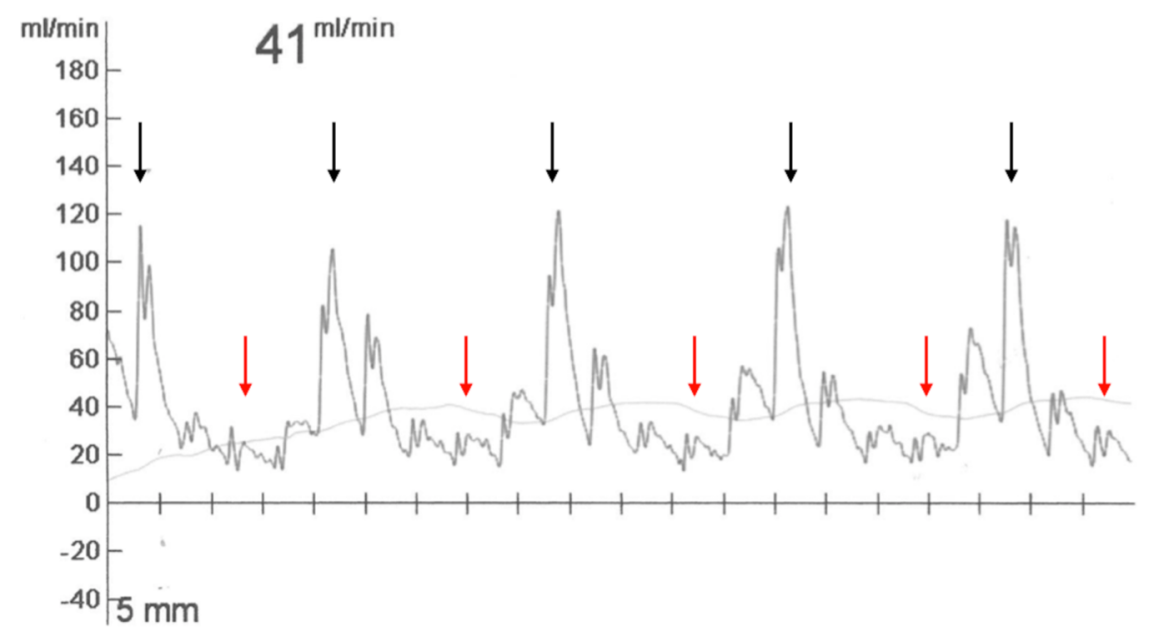

Intraoperative Doppler trace from the superior mesenteric artery. Peak systolic velocity varied between [?]120 cm/sec (black arrows) and [?]30 cm/sec (red arrows), with a periodic pattern. 


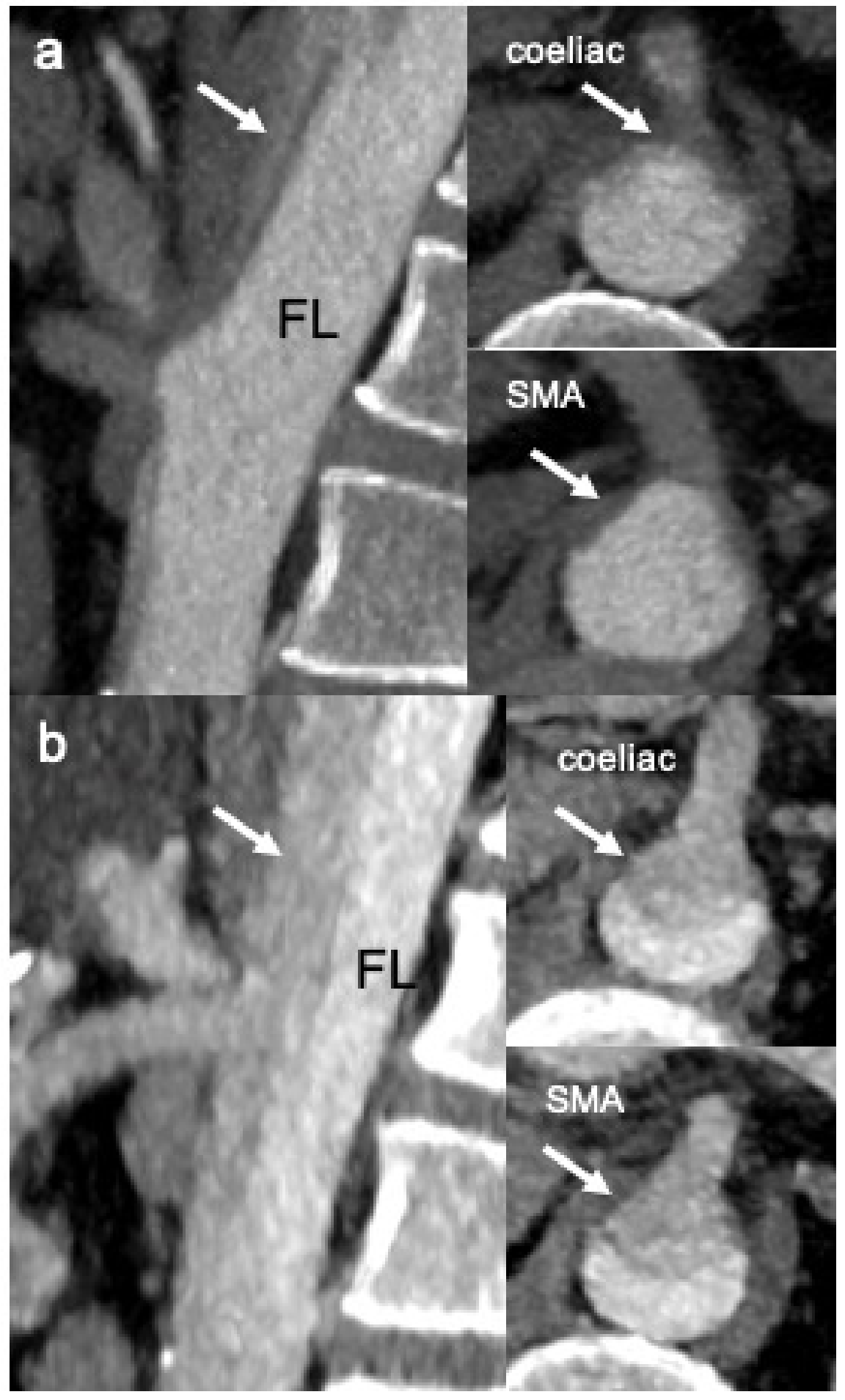




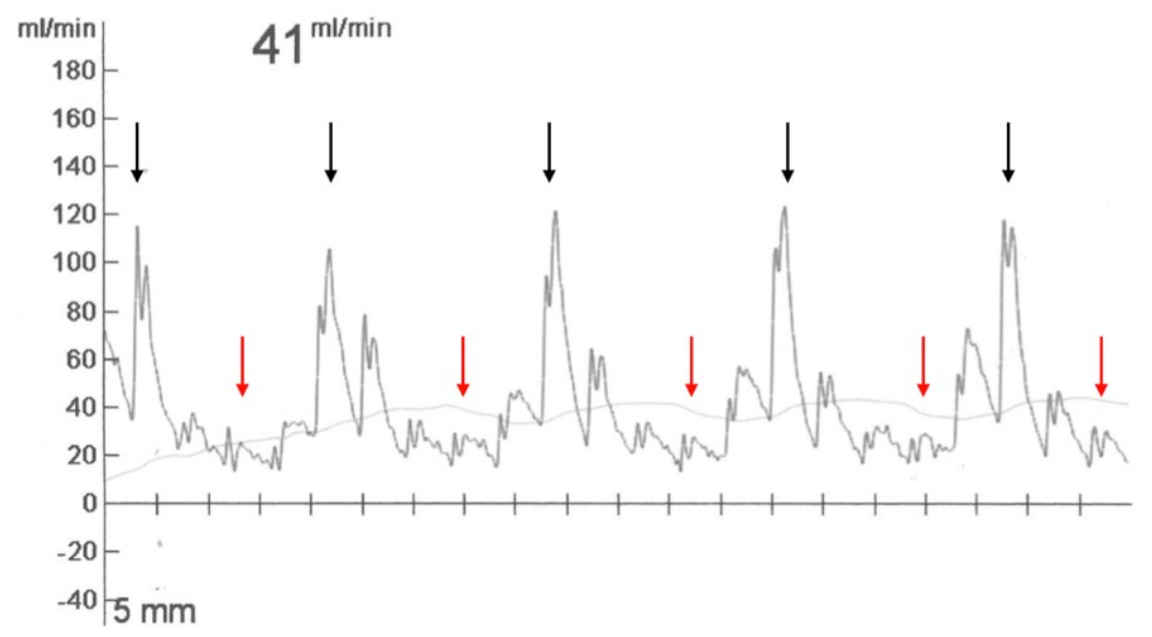

